\title{
Open Label Clinical Trial of Hydrogen Brain Food for 91 Alzheimer's Patients (Targeting Therapy for Homocysteic Acid in Blood is an Alzheimer's Cognitive Recovery)
}

Tohru Hasegawa ${ }^{{ }_{1} 1}$ and Yasubumi Uchida ${ }^{2}$

${ }^{1}$ Saga Woman Junior College, Saga 849-8440, Japan

${ }^{2}$ Saga Memorial Hospital, Takagise, 849-0917, Saga, Japan

\section{Abstract}

Introduction: Exercise and antioxidant nutrition based food are good prevention for Alzheimer's Disease. But why are these preventions effective to prevent $\mathrm{AD}$ ? Recent our findings that human $\mathrm{AD}$ patients could not excrete homocysteic acid (HA) into urine and their blood HA was a good marker for the cognitive decline in $\mathrm{AD}$, which indicated that the treatment for HA in blood can be a good therapy for the recovery of cognitive decline of $\mathrm{AD}$.

Methods: We have developed new food supplement which can compete with HA and also reduce the sulfite group of HA. We named this supplement as HBF. We observed the effect of HBF on 91 AD patients as an open labeled clinical trial.

Results: In fact we have developed the brain food which can decrease HA in blood. And we have observed that the open-labeled clinical trial of HBF (hydrogen brain food) for $91 \mathrm{AD}$ patients was good positive results. Even the final stage of $\mathrm{AD}$ patients, their cognitive behavior was surprisingly recovered.

Discussions: From our observations, it is suggested that targeting homocysteic acid in blood is a good therapy for the cognitive recovery of $\mathrm{AD}$ and also suggested that exercise and antioxidant nutrition food also can decrease $\mathrm{HA}$ in blood partially and these methods are good preventions for $\mathrm{AD}$, because normal person's HA level in blood is very low.

\author{
Publication History: \\ Received: December 06, 2016 \\ Accepted: January 10, 2017 \\ Published: January 12, 2017
}

\section{Keywords:}

Open-labeled clinical trial, Homocysteic acid in blood, Cognitive recovery

\section{Introduction}

People thinks that Alzheimer's disease (AD) is a fatal disease and if someone became $\mathrm{AD}$, his or her life will be end. There is no hope for AD. Is it a true? Why does people think so? Our scientific efforts could not succeed to clarify the true AD pathogen. At present amyloid hypothesis [1] is still strong power in AD field. However this hypothesis could not succeed to recover the AD cognitive impairment. Why? Many scientists think that the amyloid treatment was too late for $\mathrm{AD}$ patients whose neuronal damage was proceeding and amyloid treatment could not stop these damage. Is it a true? If amyloid is a true pathogen, the neuronal damage can be stopped and their cognitive ability can be recovered or can be stopped to decline.

There is a nun study [2]. From this research we have noticed that there was a big discrepancy between the pathological AD change and the brain cognitive activity. For example, sister Mary's observation. She was very active cognition in her later life, but after her death, her brain showed the typical AD brain. Why did she show so active cognition even in her AD brain? Yes, we say that her brain showed the cognitive reserve ability [3]. From these studies, we have some ideas that if we can decrease the true pathogen, we can bring back AD brain to some recovered cognitive abilities and their brains can show some cognitive reserve abilities. We have developed some brain food which could decrease HA in blood. 91 patients took this supplement for 2 months and their cognitive behaviors were measured with NM scale and we have observed the strong recovery of all patients (100\%) and even final stage of $\mathrm{AD}$ patients showed the cognitive recovery as such. From our first open-labeled results, we could recover the cognitive abilities or we could bring back the $\mathrm{AD}$ to the cognitive reserve abilities by the decreasing HA in blood.

\section{Materials and Methods}

Our open labeled clinical trial was all accepted by the ethics committee of Saga University hospital.

1. Green tea ingestion trial. 6 woman volunteers agreed this clinical trial. Green tea leaves were made into powder of $1 \mathrm{~g}$ in capsule. The volunteers ingested this capsule at every meal for 2 months. Before and after this clinical trial, their $1 \mathrm{ml}$ of blood and spot urine were collected to measure HA level. Also their MMSE score was measured before and after this clinical trial.

2. 91 patients who are all in various care houses and are suffering from Alzheimer's demenita. Male 30 (56-85years old), Female 61 (68-91 years old). Their profiles is updated in supplement data. They took our brain food, $\operatorname{HBF}$ (active Hydrogen brain food), for 2 months.

Their family agreed to take our supplement in sentence. The ethics committee of care house agreed our clinical trial.

3. NM scale: (Mental condition standard for N-type old age people) According to the NM scale, doctors or caregivers measured their patients behavior and estimated behaviour's score according topatientsbehavior. Behaviour's score were estimated by 5 items. That is, (1)housework and personal life rearrangement, (2) interest and his will (3)his conversation (4) memory (5) orientation $=$ cognitional function. Each item has 10 score. Total score is 50 .

"Corresponding Author: Dr. Tohru Hasegawa, Saga Woman Junior College ,1313 Honjomachi Oaza Honjo, Saga, Saga Prefecture 840-8440, Japan; E-mail: ghf238134@leo.bbiq.jp

Citation: Hasegawa T, Uchida Y (2017) Open Label Clinical Trial of Hydrogen Brain Food for 91 Alzheimer's Patients (Targeting Therapy for Homocysteic Acid in Blood is an Alzheimer's Cognitive Recovery). Int J Clin Res Trials 2: 111. doi: https://doi.org/10.15344/2456-8007/2017/111

Copyright: (c) 2017 Hasegawa et al. This is an open-access article distributed under the terms of the Creative Commons Attribution License, which permits unrestricted use, distribution, and reproduction in any medium, provided the original author and source are credited. 
Citation: Hasegawa T, Uchida Y (2017) Open Label Clinical Trial of Hydrogen Brain Food for 91 Alzheimer's Patients (Targeting Therapy for Homocysteic Acid in Blood is an Alzheimer's Cognitive Recovery). Int J Clin Res Trials 2: 111. doi: https://doi.org/10.15344/2456-8007/2017/111

Page 2 of 4

Total score: $0-16=$ final stage, $17-30=$ middle stage, $31-42=$ earlier stage, $43-50=$ normal

4. MMSE score: Mini Mental State Examination All patients' MMSE score were measured after 6 months HBF ingestion.

5. Our HBFsupplement:

Dr Patrick Flanagan's hydrogen powder

$: 0.05 \mathrm{~g}-0.1 \mathrm{~g}$

Coral calcium

Ferulic acid

Green tea powder

Cacao powder

Ligusticum acutilobum

Ascorbic acid

$: 0.5 \mathrm{~g}$

$: 0.6 \mathrm{~g}-1 \mathrm{~g}$

$: 0.6 \mathrm{~g}$

$: 0.6 \mathrm{~g}$

$: 0.1 \mathrm{~g}$

$: 0.2 \mathrm{~g}$

6. HA was measured by ELISA method.

Measurement of HA in blood:

\section{ELISA method}

Principle of the Assay: The microtiter plate provided in this kit has been pre-coated with an antibody specific to Hcy. Standards or samples are then added to the appropriate microtiter plate wells with a biotin-conjugated antibody preparation specific for Hcy and Avidin conjugated to Horseradish Peroxidase (HRP) is added to each microplate well and incubated. Then a TMB $\left(3,3,5,5^{\prime}\right.$ tetramethylbenzidine) substrate solution is added to each well. Only those wells that contain Hcy, biotin-conjugated antibody and enzyme-conjugated Avidin will exhibit a change in color. The enzyme-substrate reaction is terminated by the addition of a sulphuric acid solution and the color change is measured spectrophotometrically at a wavelength of $450 \mathrm{~nm}$ $+/-2 \mathrm{~nm}$. The concentration of Hcy in the samples is then determined by comparing the O.D. of the samples to the standard curve. (the copy from MyBioSource co.)

\section{Results}

\section{The effect of green tea leaves on MMSE score and HA in blood}

It is well-known that green tea can prevent Alzheimer disease (1), then we are interested in the healing-like effect of green tea on the cognition. 6 Alzheimer's dementia patients in one care house agreed the ingestion of green tea leaves for every meal. They took this green tea leaves for 2 months. Before and after 2 months, their MMSE score and HA in blood measured.

6 Alzheimer's patients were all female. Their ages were $77,82,86,87$, 91, 91. Green tea powder induced MMSE change and their blood HA change. The results were shown in Figure 1. 3 patients showed the decrease change in blood HA (number 1,2,5), but two patients showed the increase (number 3,4) and 1 patient showed no change (number 6).

On the other hand, their MMSE score change were as follow: Number $1: 6$ to 9 , Number 2: 8 to 10 , Number 3: 16 to 14 , Number 4: 20 to 16 Number 5: 19 to 22 , Number 6: 24 to 26 .

The relationship between the blood HA change and MMSE score change. The result is shown in Figure 2. Clearly shown in Figure 2, the relationship between blood HA change (percent change) and MMSE score change is strong negative statistical significant $(r=-0.96$, $\mathrm{p}=0.00018, \mathrm{n}=6$ ).

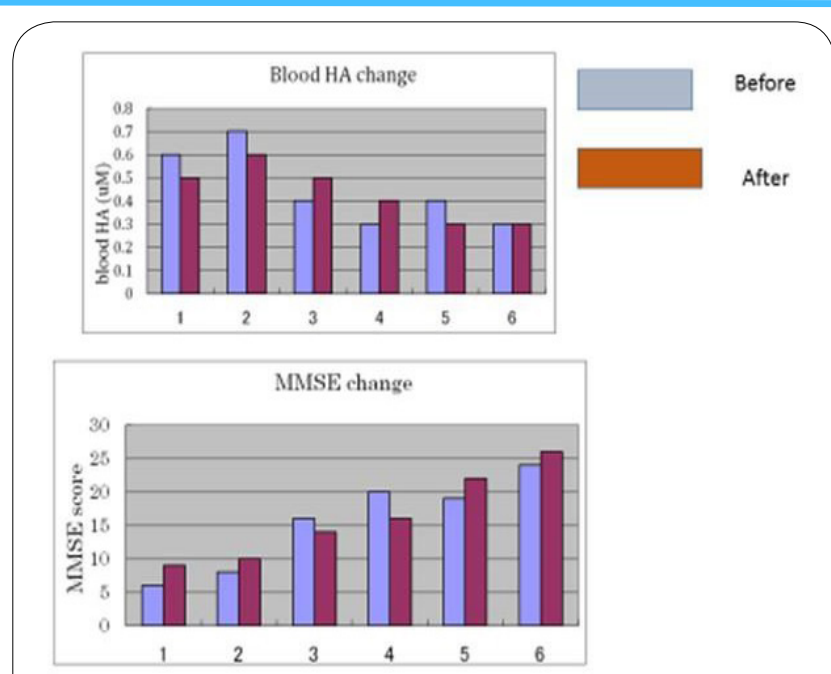

Figure 1: The effect of green tea leaves on MMSE score and HA inblood.

Before: before ingestion of green tea powder. After: 2 months later after the ingestion of green tea powder.

Note: Number shows the Alzheimer's patient. 1: age 82, 2: age 77, 3: age 91, 4: age 87,5 : age 91,6 : age 86 .

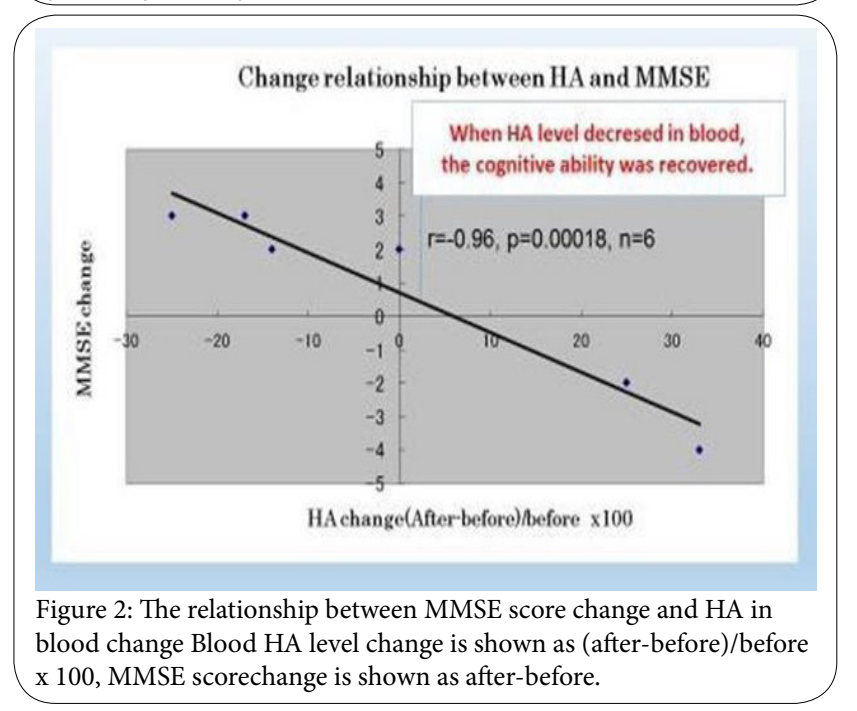

\section{Open-labeled clinical trial of $91 \mathrm{AD}$ patients}

From 1 experiment, we understood that HA in blood was related to MMSE score. In other words, HA in blood is suggested to control MMSE score, or HA in blood may control the cognitive ability. If we can develop some supplement to decrease HA in blood, the cognitive recovery can be gotten in $\mathrm{AD}$ patients. First we observed the effect of our supplement on HA in blood. First 8 male volunteers who are suffering from memory problem and whose ages were 65-75 years old took our supplement for 2 months. Their memory problem were all disappeared and their blood HA were measured. The results are shown in Figure 3 Clearly shown in Figure 3, their blood HA were decreased after 2 months ingestion of our supplement.

From this observation, we conducted the open-labeled clinical trial of $91 \mathrm{AD}$ patients. The results are shown in Figure $4.100 \%$ all patients increased their NM scores. These all date are summarized with statistically analysis. The result is shown in Table 1 (supplementary files). The results were really amazing. Even final stage of patients showed the recovery process as such. However they were still suffering 


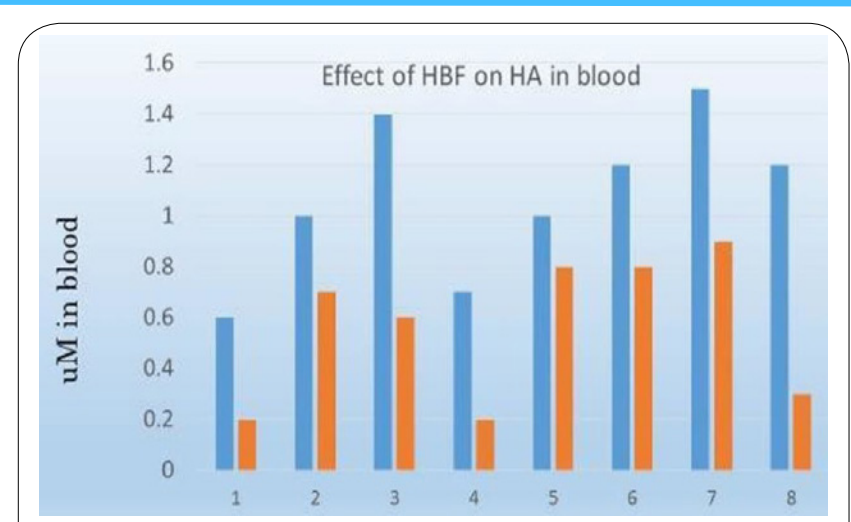

Figure 3: Effect of HBF on blood homocysteic acid level. HA in blood was measured by ELISAmethod.

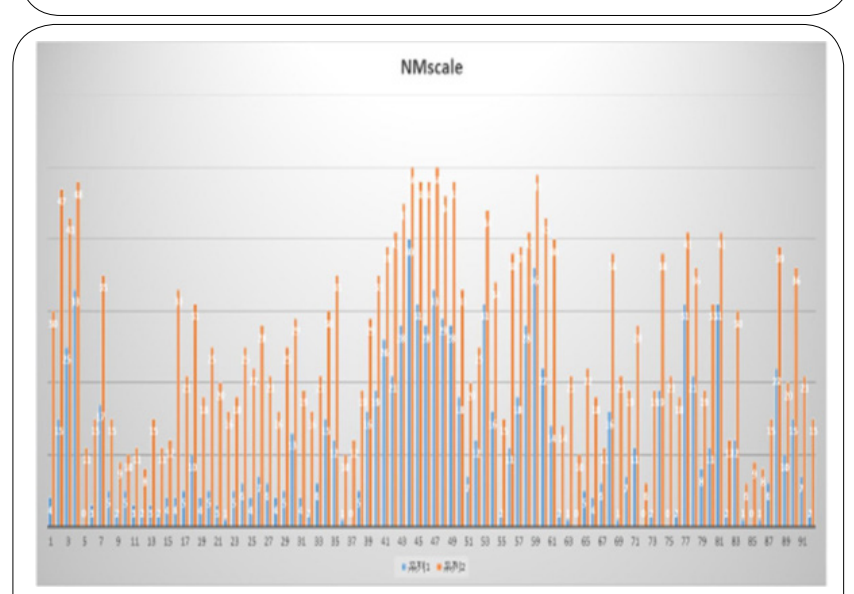

Figure 4: Open-labeled clinical trial of 91 ADpatients.

All patients were checked by doctors and we got their agreements for assessment of their behaviors by NM scale; Blue bar indicates before $\mathrm{HBF}$ ingestion and yellow bar indicates 2 months later HBF ingestions; Score:0-16: final stage, 17-30:middle stage, 31-42 early stage, 43-50 normal

from memory problem. But they could understand the conversation, they could greet the caregivers. They did not recognize their families, but after taking supplement, they could recognize their families. Some middle stage of $\mathrm{AD}$ patients, especially case 4 , his cognitive recovery was perfect and he completely recovered to normal.

Figure 5 shows the MMSE score of all patients. Final stage patients did not increase their MMSE score. All final patients showed 0 score.

\section{Discussion}

\section{The effect of green tea leaves on MMSE score and HA inblood}

We were interested in the healing-like effect of green tea on the cognition of AD. Unfortunately this green tea healing-effect was transient. This healing- effect could not observed constantly. That is, after 3 months, this healing- effect was disappeared. Maybe green tea induced the drug-metabolizing enzyme.

We observed the relationship between blood HA level and MMSE score. And Figure 2 shows the very strong perfect relationship between blood HA level change and MMSE score change. From Figure 2 result, we can say that

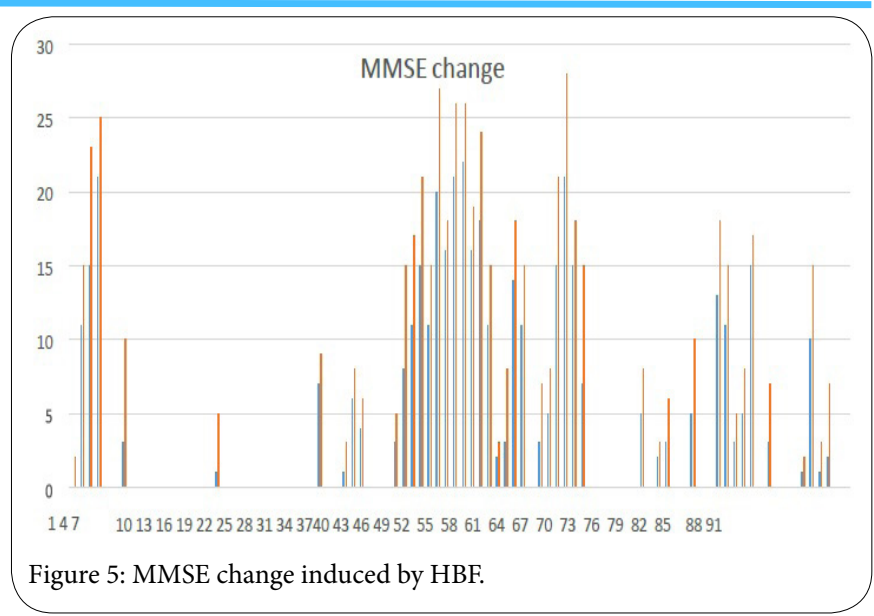

blood HA can control the MMSE score change. That is, blood HA can control the cognitive ability in Alzheimer's patient.

Why can the blood HA control cognitive ability inpatients?

First HA is a glutamate neurotransmitter and HA itself can induce neurodegeneration at a higher level with no amyloid (4), and the HA toxicity induces a calcium influx (5) or a mitochondrial inhibition (6) or oxidative stress (4) to bring the neuronal disfunction.

NMDA receptor in blood brain barrier (BBB) can control its permeability $[7,8]$. Then the blood HA can increase the permeability of $\mathrm{BBB}$ and $\mathrm{HA}$ can pass through $\mathrm{BBB}$ and enters into hippocampus, because hippocampus's BBB is very thin [9]. And entered HA can interact with already accumulated amyloid beta and HA increases its neurotoxicity, because amyloid beta increases HA toxicity (unpublished observation).

In a study published in Nature [10,11], Stanford University School of Medicine scientists have found substances in the blood of old mice that makes young brains act older. These substances, whose levels rise with increasing age, appear to inhibit the brain's ability to produce new nerve cells critical to memory and learning.

The findings raise the question of whether it might be possible to shield the brain from aging by eliminating or mitigating the effects of these apparently detrimental blood-borne substances.

APOE4 is the gene involved in the mechanism of development of AD. APOE4 increases the permeability of Blood Brain Barrier (BBB) [12], which allows the pathogen in the peripheral blood to pass BBB and disturb the brain functions. This report indicates the possibility of APOE4 early-onset gene.

Finally, the third report [13] describes an actual early patient of $\mathrm{AD}$ showing the destruction of hippocampal BBB. However, these reports did not describe which pathogen in the blood is involved and what resulted in an increased BBB permeability in aged hippocampus.

It has been reported that homocysteic acid (HA) is the probable pathogen of $\mathrm{AD}$ in blood [14-17], and it increases $\mathrm{BBB}$ permeability by NMDA receptor activation [18]. Especially it is interesting that HA can induce the amyloid $\beta-42$ which accumulated into neuronal cell [17]. HA is known as a glutamate receptor agonist [19]. JAD (14) study reported a significant negative relationship between MMSE scores and HA levels in blood. Thus, HA can be one of the pathogens in blood. 
Citation: Hasegawa T, Uchida Y (2017) Open Label Clinical Trial of Hydrogen Brain Food for 91 Alzheimer's Patients (Targeting Therapy for Homocysteic Acid in Blood is an Alzheimer's Cognitive Recovery). Int J Clin Res Trials 2: 111. doi: https://doi.org/10.15344/2456-8007/2017/111

Page 4 of 4

\section{Open-labeled clinical trial}

First we should give comments about our supplement.

Our HBF supplement contained Ferulic acid (FA). This FA chemical structure is very similar as that of $\mathrm{HA}$, which can compete $\mathrm{HA}$ at NMDA receptor of BBB. In fact the uptake competition between HA and FA of $10 \mathrm{uM}$ in cultured cell of the mouse fetus brain was observed (unpublished observation). Also HBF produced the hydrogen gas in blood which could reduce the sulfite group of HA to homocysteine. In fact the some patients showed the decrease in HA was observed parallel to the increase in homocysteine in blood.

Second we should give comment for double-blind clinical trial for the final stage of $\mathrm{AD}$ patients. They cannot understand what happened in their world. In other words, I am sorry to say so, they are almost experimental animal, which can not induce the placebo effect. Also caregivers and doctors also observed the completely their qualitative change of behavior. For example, one patients showed all day comma, but after taking our HBF, they showed awake and said something to neighbors. So in case of final stage of patients, they needed not double-blind test.

Now we can present the clinical trial of brain food which can destroy blood HA on the recovery of cognitive impairment ofAD patients. The results are very impressive. Even the final stage of patient could recover their cognitive behavior, could understand his or her families, and finally could understand the conversations with others. However they still suffered from memory problem. In fact all final stage of patients showed no increasing of MMSE score. All final patients showed 0 score before and after the HBF ingestion. Because the hippocampal structure of a final stage of patients would be destroyed and HBF supplement could not recover the hippocampal structure. From Figure 5, it is clear that MMSE scale measurement is not better than NM scale, because at even MMSE score 0 , patients showed the recovery process of NM scale. Because the some cognitive behavior is not dependent on the hippocampal function [20].

However middle stage of patients increased their MMSE score, because their hippocampal activities could be recovered and consequently their MMSE score increased compared with before one.

Our clinical trial can offer the some hopes in $\mathrm{AD}$ field. That is, the cognitive impairment of $\mathrm{AD}$ can be recovered by the adequate treatments such as brain food or drugs so on. These adequate treatments should destroy the possible pathogens in blood such as HA or beta2-microglobulin. Then this adequate treatment can recover the cognitive reserve abilities of $\mathrm{AD}$ patients. Because it knows that the peoples with even Alzheimer's pathological structure's brain can show the normal brain works, and it says that this is cognitive reserve ability.

So we present the hope that the adequate treatments for $\mathrm{AD}$ can bringback their cognitive reserve abilities.

\section{Acknowledgement}

The authors thank their gratitudes to Nurse Nanri in Saga memorial hospital for her devoted care to the patients (Figure 4 case 9-14).

\section{Competing Interests}

The authors declare that they have no competing interests.

\section{References}

1. Karran E, Mercken M, De Strooper B (2011) The amyloid cascade hypothesis for Alzheimer's disease: an appraisal for the development of therapeutics. Nat Rev Drug Discov 10: 698-712.

2. Snowdon DA (1997) Aging and Alzheimer's Disease: Lessons From the Nun Study. Gerontologist 37: 150-156.

3. Stern $Y(2012)$ Cognitive reserve in ageing and Alzheimer'sdisease. Lancet Neurol 11: 1006-1012.

4. Lockhart B, Jones C, Cuisinier C, Villain N, Peyroulan D, et al. (2000) Inhibition of L-homocysteic acid and buthionine sulphoximine-mediated neurotoxicity in rat embryonic neuronal cultures with a-lipoic acid enantiomers. Brain Res 855: 292-297.

5. Shankar GM, Bloodgood BL, Townsend M, Walsh DM, Selkoe DJ, et al. (2007) Natural Oligomers of the AlzheimerAmyloid-s Protein Induce Reversible Synapse Loss by Modulating an NMDA-Type Glutamate Receptor- Dependent Signaling Pathway. J Neurosci 27: 2866-2875.

6. Folbergrová J, Jesina P, Drahota Z, Lisý V, Haugvicová R,et al. (2007) Mitochondrial complex linhibitionincerebralcortexof immature rats following homocysteic acid-inducedseizures. Exp Neurol 204: 597-609.

7. Ramos Coutinho M, da Silva LF, Menescal-de-Oliveira L (2008) Modulation of tonic immobility in guinea pig PAG by homocysteic acid, a glutamate agonist. Physiol Behav 94: 468-473.

8. Beard RS Jr, Reynolds JJ, Bearden SE (2011) Hyperhomocysteinemia increases permeability of the blood-brain barrier by NMDA receptordependent regulation of adherens and tight junctions. Blood 118: 20072014.

9. Vorbrodt AW, Dobrogowska DH, Ueno M, Tarnawski M (1995) A quantitative immunocytochemical study of blood-brain barrier to endogenous albumin in cerebral cortex and hippocampus of senescence-accelerated mice (SAM). Folia Histochem Cytobiol 33: 229-237.

10. Scudellar M (2015) By splicing animals together, scientists have shown that young blood rejuvenates old tissues. Now, they are testing whether it works for humans. Nature 517: 426-429.

11. Villeda SA, Luo J, Mosher KI, Zou B, Britschgi M, et al. (2011) The ageingsystemic milieu negatively regulates neurogenesis and cognitivefunction. Nature 477: 90-4.

12. Bell RD, Winkler EA, Singh I, Sagare AP, Deane R, et al. (2012) Apolipoprotein E controls cerebrovascular integrity via cyclophilin A. Nature 485: 512-516.

13. Montagne A, Barnes SR, Sweeney MD, Halliday MR, Sagare AP, et al. (2015) Blood-Brain Barrier Breakdown in the Aging Human Hippocampus. Neuron 85: 296-302.

14. Hasegawa T, Ichiba M, Matsumoto SE, Kasanuki $K$, Hatano $T$, et al. (2012) Urinary Homocysteic Acid Levels Correlate with Mini-Mental State Examination Scores in Alzheimer's Disease Patients. J Alzheimer's Dis 31 59-64.

15. Hasegawa T, Mikoda N, Kitazawa M, LaFerla FM (2009) B6 deficient feeding or homocysteic acid induces the earlier Alzheimer's pathological change in normal C57BL male mice. Nature Precedings.

16. Hasegawa T, Mikoda N, Kitazawa M, LaFerla FM (2010) Treatment of Alzheimer's disease with anti-homocysteicacid antibody in 3xTg-AD male mice. PLoS One 5: e8593.

17. Hasegawa T, Ukai W, Jo DG, Xu X, Mattson MP, et al. (2005) Homocysteic acid induces intraneuronal accumulation of neurotoxic Abeta42: Implication for the pathogenesis of Alzheimer's disease. J Neurosci Res 80: 869-876.

18. Miller RD, Monsul NT, Vender JR, Lehmann JC (136) NMDA- and endothelin-1-induced increases in blood-brain barrier permeability quantitated with Lucifer yellow. J Neurol Sci 136: 37-40.

19. Do KQ, Herrling PL, Streit P, Cuenod M (1988) Release of neuroactive substances: homocysteic acid as an endogenous agonist of the NMDA receptor. J Neural Transm 2: 185-190.

20. Eichenbaum H (2004) Hippocampus: Cognitive Processes and Neural Representations that Underlie Declarative Memory. Neuron 44: 109-122. 